\title{
Circle Detection Using a Gabor Annulus
}

\author{
Adam Rhodes \\ aqr@cs.nott.ac.uk \\ Li Bai \\ bai@cs.nott.ac.uk
}

School of Computer Science

University of Nottingham,

Nottingham, UK

\begin{abstract}
We present a novel circle detection technique based on the desirable properties of Gabor wavelet filters. We describe the technique and its Gabor wavelet origins, and perform multiple experiments using both synthetic images and real microscopic images of red blood cells. Our experiments show the proposed technique's ability to detect single and overlapping circles, with improved results over traditional techniques including both the Circular Hough Transform and its modifications which include edge orientation. The improved results are demonstrated under high levels of noise and for partially defined overlapping circles.
\end{abstract}

\section{Introduction}

This paper introduces a new method for circle detection based on the properties of Gabor wavelet filters. Circles are found frequently in nature, as perfect circular structures provide an optimal area-to-perimeter ratio. The ability to accurately detect circles is therefore useful in a range of practical image processing applications. One common application is to automatically count cells in biological experiments $[\square, \square]$, providing quantitative results as experimental parameters are varied. This process may be infeasibly time consuming when counting cells manually for large numbers of experiments $[\theta, \mathbb{Q}]$. A further more advanced application is the tracking of cancer or stem cells over time [ $\boldsymbol{Q}, \mathbf{Q}]$, as understanding the movement, lineage and therefore differentiation of these cells as they grow into some specific tissue is vital to the understanding of these biological processes. The tracking of thousands of individual cells via reliable circle detection again provides quantitative data that would previously have been either impractical or extremely time consuming to gather manually. A recent review of cell tracking techniques is found in []].

It is also known that the recognition of radially symmetric features is a preattentive process in the natural visual system which can drive the recognition of salient visual features $[\square, \square]$. It is the radial symmetry of circular image features that we hope to exploit, and this motivates the design of our proposed circle detection method. In the case of circular features identified by strong edges, techniques such as the Circular Hough Transform (CHT) can be used to identify the circle's centre location. However in real applications it is common to encounter circular features where edges are not clear, and some radial pattern may instead identify the feature. In addition image noise and imperfect symmetry may contribute to a circular feature that is difficult to detect using existing edge based techniques.

(c) 2011. The copyright of this document resides with its authors. BMVC $2011 \mathrm{http}: / / \mathrm{dx} . \mathrm{doi} . \mathrm{org} / 10.5244 / \mathrm{C} .25 .108$ It may be distributed unchanged freely in print or electronic forms. 


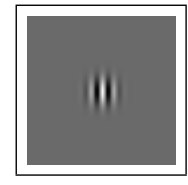

$s=1$

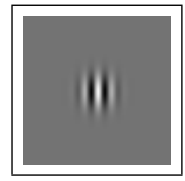

$s=1.2$

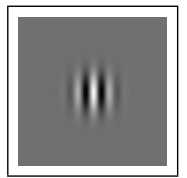

$s=1.4$

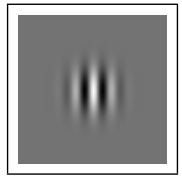

$s=1.6$

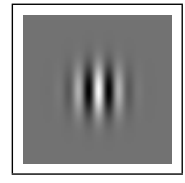

$s=1.8$

Figure 1: Example of Gabor wavelet filters at 5 scales for a single orientation. Filters were generated using Equation 1 with $\sigma_{x}=10, \sigma_{y}=10, f_{0}=1 / 5$ and a scale factor $s$ (shown).

We will first introduce existing circle detection techniques, and present an overview of Gabor wavelet filtering and its traditional uses. In Section 2 the proposed technique is detailed, leading to a number of experiments using both synthetic and real medical images which are described in detail in Section 3. Our conclusions are then presented in Section 4.

\subsection{Relevant literature}

The CHT [ $\mathrm{Q}]$ is widely used as a circle detection technique. The CHT typically relies on an edge detector to provide candidate edge points for circles within the image. The CHT then iterates through each of these edge points, and for each point assigns a circle of a specific radius to an accumulator space. This output accumulator space will contain peaks where numerous circles (originating at edge points) overlap, indicating the likely centre location. This CHT can be implemented as a simple convolution operator [ $[$ ] , by creating a convolution filter containing a circle of the desired radius. The convolution output then performs as the accumulator space for this radius, with peaks indicating circle centre locations.

Work by Atherton and Kerbyson [ $[$ ] focussed on assessing the CHT and modifications to the technique. This includes an 'Annulus' version of the CHT which uses a single accumulator space for multiple radii, and a Phase Coded Orientation Annulus (PCOA) which incorporates edge orientation. Other recent modifications to the CHT include ideas such as the use of optical flow information in video sequences [ㅁ] , and modifications to distinguish circles from arc segments [D]. Related techniques which exploit radial symmetry via oriented voting kernels are proposed in [ㅁ] and [ष]].

\subsection{Gabor wavelet filters}

Gabor wavelet filters are used in a large number of image processing tasks, for example in

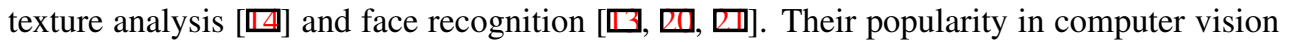
stems from the natural basis for Gabor wavelets, which have been found to be similar to the receptive fields of simple cells within the primary visual cortex of mammals [ $\mathrm{\theta}]$.

The traditional Gabor wavelet filter is a complex plane wave restricted by a Gaussian envelope, and is defined as follows [ㅁ, 미:

$$
G(x, y)=\frac{1}{2 \pi \sigma_{x} \sigma_{y}} e^{-\pi\left[\frac{\left(x-x_{0}\right)^{2}}{\sigma_{x}^{2}}+\frac{\left(y-y_{0}\right)^{2}}{\sigma_{y}^{2}}\right]} e^{i\left[2 \pi f_{0} x\right]}
$$

The first exponent is an elliptical Gaussian, with standard deviation $\sigma_{x}$ in the waveform direction, and $\sigma_{y}$ in the perpendicular direction. The second exponent is the complex plane wave with frequency $f_{0}$ in the $\mathrm{x}$ direction. $\left(x_{0}, y_{0}\right)$ specify the centre coordinates of the filter in the spatial domain. 


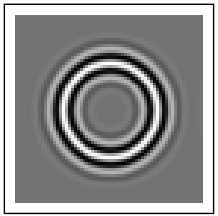

(a)

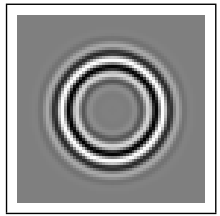

(b)

Figure 2: Example Gabor Annulus filter. (a) Real part of filter (b) Imaginary part of filter. The filters were generated using Equation 2 with $r_{0}=16, \sigma=10$ and $f_{0}=1 / 5$.

The filter defined in Equation 1 can be treated as a 'mother wavelet', which can be scaled and rotated to produce a family of filters. These filters, applied to an input image via convolution, will each produce a maximum response in areas of an image containing features or patterns which match their specific scale and orientation. Therefore in most tasks a range of filters covering expected scales and orientations of underlying image features are generated. The use of 5 scales and 8 orientations is common [四] - resulting in a total of 40 filters. Example Gabor wavelets are shown in Figure 1. Work by Jain and Farrokhnia [ $[$ ] has addressed the topic of optimal parameter selection for Gabor filters, in order to obtain good coverage with minimal overlap in the spatial-frequency domain.

Specifically, Gabor wavelet filters are a method of extracting the spatial location of underlying frequencies within an image. Each filter provides a maximal response in areas of an image where there is an underlying pattern or image feature with the same frequency characteristics as the filter, at the same scale and orientation. It is this useful property which acts as the basis for our new filter design aimed at extracting circular or symmetrical features within an image, where clear edges may be absent or only partially present. The ability of Gabor wavelets to respond strongly to more general image features based on underlying textures and frequencies is key to the design of our proposed Gabor Annulus technique.

A variation of Gabor wavelets where the waveform direction is modified to extend outwards from the centre is the Circular Gabor Filter (CGF), first introduced in work by Zhang et al. [ $\square$ ] with applications in texture segmentation, and later in eye location tasks [ $\square$ ]. The circular symmetric nature of the filter was found to be useful for obtaining a rotation invariant response to textures.

\section{Gabor Annulus for Circle Detection}

Our proposed filter aims to use the ability of Gabor wavelets to detect image features and patterns at specific scales and orientations in order to detect circular features. The proposed Gabor Annulus technique therefore offsets the traditional Gabor filter by a radius which wraps around the origin, and is defined as follows:

$$
G(x, y)=\frac{1}{2 \pi \sigma r_{0}} e^{-\pi\left[\frac{\left(r-r_{0}\right)^{2}}{\sigma^{2}}\right]} e^{i\left[2 \pi f_{0}\left(r-r_{0}\right)\right]}
$$

where

$$
r=\sqrt{\left(x-x_{0}\right)^{2}+\left(y-y_{0}\right)^{2}}
$$

In this new filter $\sigma$ specifies the standard deviation of the Gaussian envelope in the waveform direction, which now expands radially outwards from the centre of the filter. $r_{0}$ specifies 
the radius of the 'annulus' shape. The second exponent is the complex plane wave with frequency $f_{0}$ in the waveform direction. $\left(x_{0}, y_{0}\right)$ specify the centre coordinates of the filter in the spatial domain.

An example of a generated Gabor Annulus filter is provided in Figure 2. The filter is similar to the original Gabor wavelet filter, but the complex plane wave now radiates from the centre of the filter (similarly to the CGF discussed earlier), and the Gaussian envelope is now centred around the radius of the filter. This creates a filter that responds to image features which are circular in form and consist of matching radial frequencies on their boundaries. The filter response will be strongest at the centre of a matching feature, giving a centre location for the circular image feature. Similarly to traditional Gabor filters the new Gabor Annulus technique can use a single 'mother' filter to create a family of filters at various scales. These can then be used to match circular image features at various sizes within the image. As the filter is radially symmetric there is no need to also generate rotations at different orientations as in traditional Gabor wavelet filtering.

\section{Experiments}

\subsection{Tests using synthetic images}

In order to demonstrate the proposed Gabor Annulus technique as a viable circle detection method, we have first performed a number of experiments using synthetic images. Work by Atherton and Kerbyson [⿴囗十 used synthetic images to compare the CHT technique with common modifications, and their own proposed method. We have therefore adopted the same approach and implemented these methods for comparison, and tested our own proposed method against these techniques. The methods implemented were the standard CHT, the CHT Annulus which covers a small range of radii in a single filter, and the Phase Coded Orientation Annulus (PCO Annulus or PCOA) which incorporates both edge orientation information and a phase coding to detect circles over a specific range of radii. Detailed descriptions of the construction of these filters (implemented as convolution operators) can be found in [四, where it was demonstrated that the PCOA technique performs strongly as a circle detector, including in the presence of noise.

\subsubsection{Single circle}

A synthetic test image of dimensions $128 \times 128$ pixels, containing a single circle of radius 16 pixels was created, with the circle centred in the image. Background values are assigned the value 0 , with pixels belonging to the circle assigned the value 1 . The single circle test image is shown in Figure 3 (a). All of the techniques to be tested require a radius parameter, which was set to 16 pixels to match the test image. In the case of the Annulus and PCOA techniques a minimum and maximum radius are required, and these were set to 8 and 24 pixels respectively to match the values used in [四]. The proposed Gabor Annulus filters were generated using Equation 2 with the parameters $r_{0}=16, \sigma=10$ and $f_{0}=1 / 5$. As the Gabor Annulus filters are complex, the absolute values of the convolution output are used, as is typically the case in traditional Gabor wavelet filtering.

All operators are applied to an edge image, which is computed from the test image using a Sobel edge detector. This edge image therefore contains the outline of a single circle. As our proposed technique does not depend on edge information it is tested both against the 


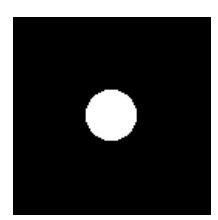

(a)

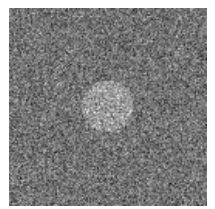

(d)

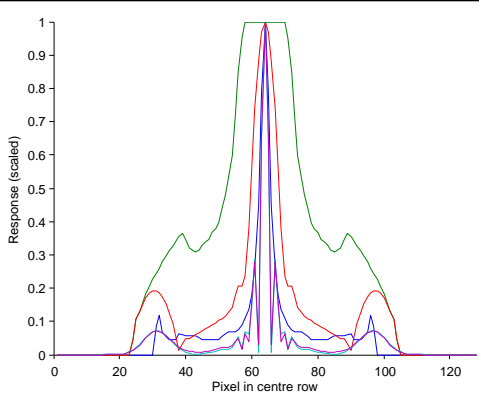

(b)

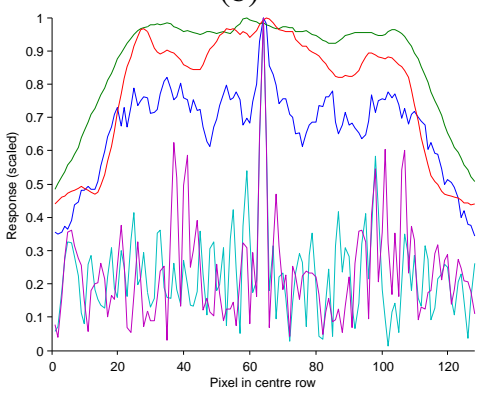

(e)

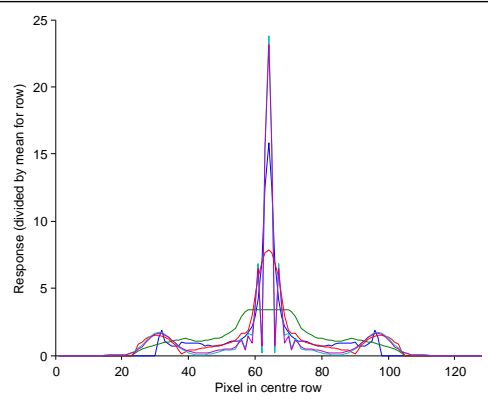

(c)

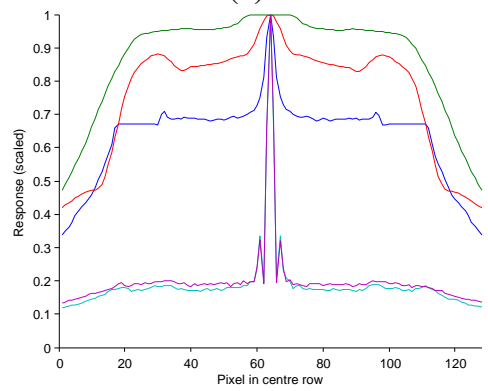

(f)

- Circle Hough Transform — Annulus — PCO Annulus — Gabor Annulus —— Gabor Annulus (Direct)

Figure 3: Tests with a single circle image. Graphs all show filter responses at each pixel for the centre row of the image. (a) Single circle test image (b) Filter responses (scaled by the maximum response in the row for each filter). (c) Filter responses divided by the mean filter response for the row. (d) Single circle test image with added Gaussian noise $(\sigma=0.7)$ (e) Filter responses against noisy image. (f) Average filter responses at each pixel repeating the test with random Gaussian noise 1000 times.

same edge image as the other operators, and also against the original test image directly. In order to assess the operator's respective performance in more practical scenarios the test image is altered to include added noise. Zero-mean white gaussian noise was added to the test image, with deviation $\sigma=0.7$ ( $\mathrm{SNR}=1.4)$. A resulting test image with added noise is shown in Figure 3 (d).

Results against the single circle test image are shown in Figure 3. Each graph shows a cross section of the filter response, taken across the centre row of the image. This allows a comparison of the filter responses for each technique, where a single thin peak indicating the circle's centre location is the ideal result, ideally with a low filter response elsewhere in the row. Figure 3 (b) shows the responses against the single circle (without noise) test image, and as expected all methods perform well in this simple case. Figure 3 (c) presents the same results, but instead of scaling the results by peak response the responses are divided by the mean response for the row. Therefore in this graph a high peak to mean value is desired, with both of the proposed Gabor techniques showing the best results. As the peak to mean ratio is a useful indicator of a technique's peak response performance compared to the level of background response noise, the ratios for each experiment are included in Table 1.

Figure 3 (e) shows the scaled responses when tested against the noisy image. In this case 


\begin{tabular}{|l|c|c|c|c|}
\cline { 2 - 5 } \multicolumn{1}{c|}{} & \multicolumn{3}{c|}{ Peak to mean response ratios for synthetic test images } \\
\hline \multirow{2}{*}{ Technique } & $\begin{array}{c}\text { Single } \\
\text { Circle }\end{array}$ & $\begin{array}{c}\text { Single }+ \\
\text { Noise }\end{array}$ & $\begin{array}{c}\text { Multi } \\
\text { Circle }\end{array}$ & $\begin{array}{c}\text { Multi + } \\
\text { Noise }\end{array}$ \\
\hline CHT & 15.79 & 1.50 & 5.20 & 1.52 \\
Annulus & 3.43 & 1.15 & 1.69 & 1.16 \\
PCOA & 7.85 & 1.28 & 2.66 & 1.30 \\
Gabor Annulus & $\mathbf{2 3 . 8 1}$ & $\mathbf{4 . 3 6}$ & 8.25 & $\mathbf{4 . 2 4}$ \\
Gabor Annulus (Direct) & 23.17 & 4.19 & $\mathbf{8 . 2 6}$ & 4.17 \\
\hline
\end{tabular}

Table 1: Peak response to mean response ratios for synthetic circle images. The best results for each test are shown in bold. A high peak response (indicating circle centre) against a low background mean response is desirable, resulting in a high peak to mean response ratio. The circle detection techniques and test images used are detailed in Section 3.1.

it is apparent that the traditional techniques perform poorly, with only the CHT showing a peak indicating the circle's centre location, but with comparatively high response throughout the entire row. The proposed technique again performs better even in this high level of noise, with a single thin peak against a lower background response for the rest of the row.

As the noise added to the test image is random, the test was repeated 1000 times with a new test image generated for each experiment. Figure 3 (f) then shows the results of these tests, showing mean responses at each pixel location for the 1000 trials. This again demonstrates that the proposed technique shows a higher peak response compared to the background response for the rest of the row. It is, however, also observed that the comparison of this repeated test with the single result shown in Figure 3 (e) highlights a high deviation for the background response in our proposed technique.

Table 1 shows the peak response to mean response ratio for each synthetic test image, with the proposed Gabor Annulus techniques performing best in all tests.

\subsubsection{Multiple overlapping circles}

The single circle tests were then extended to include multiple overlapping circles (a scenario that was not covered in [四]). In addition to the single circle of radius 16 pixels centered at the centre of the image, two additional circles were added of the same size and also located on the central row of the image, but offset by 24 pixels to the left and right of the existing circle respectively. The resulting test image, which now contains only partially overlapping circles, can be seen in Figure 4 (a). Zero-mean white gaussian noise was again added to the test image $(\mathrm{SNR}=1.4)$, shown in Figure $4(\mathrm{~d})$.

The results for multiple circles are shown in Figure 4. In these tests we now expect to see three distinct peaks in the output responses (at pixels 40,64 and 88 respectively), due to the presence of 3 overlapping circles. This is of course a more difficult test even in the no-noise case, as the overlapping circles are not complete and lack significant portions of their edges.

Figure 4 (b) shows the results for the noise free image, with all except the Annulus technique highlighting three peaks at the correct locations. The proposed Gabor techniques again demonstrate a consistently lower background response for the row, which is highlighted in Figure 4 (c) which shows peak to mean ratios.

Figure 4 (e) shows the multiple circle result when testing with added noise, where only the $\mathrm{CHT}$ and proposed techniques are able to show three distinct peaks at circle centres. The 


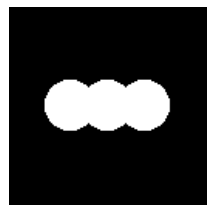

(a)

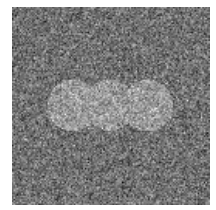

(d)

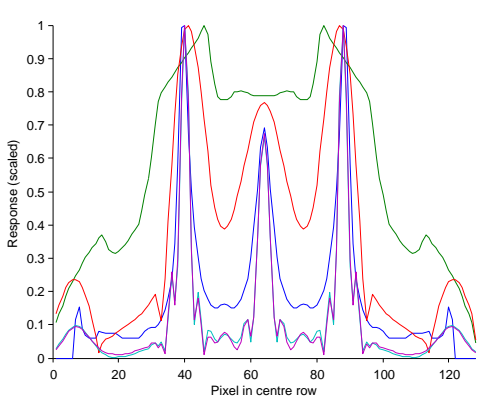

(b)

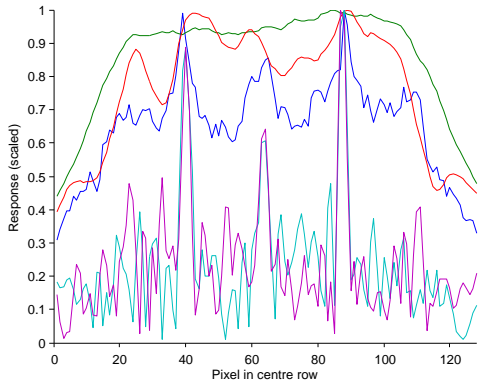

(e)

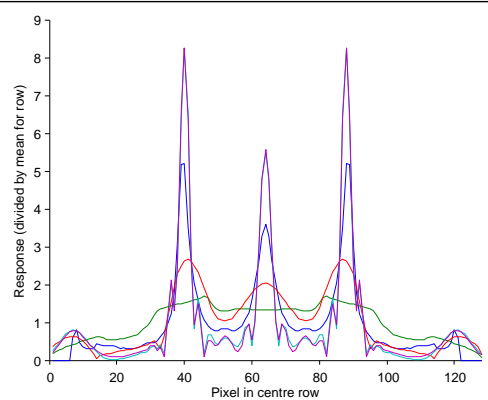

(c)

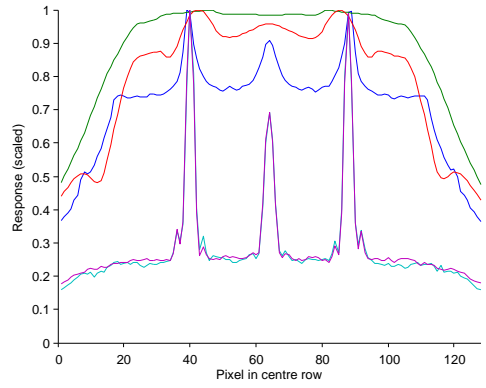

(f)

Figure 4: Tests with a multiple circle image. Graphs all show filter responses at each pixel for the centre row of the image. (a) Multiple circle test image (b) Filter responses (scaled by the maximum response in the row for each filter). (c) Filter responses divided by the mean filter response for the row. (d) Multiple circle test image with added Gaussian noise $(\sigma=0.7)$ (e) Filter responses against noisy image. (f) Average filter responses at each pixel repeating the test with random Gaussian noise 1000 times.

CHT again shows a comparatively high background response level, with the proposed Gabor Annulus techniques showing a lower background response. Again repeating the process for the single circle tests, this noisy test was repeated 1000 times with a new test image generated for each experiment. Figure 4 (f) shows the results of this test, and again demonstrates three clear peaks against a lower mean background response for the proposed Gabor Annulus techniques. Table 1 also shows the peak response to mean response ratio for the multiple circle test images, with the proposed Gabor Annulus techniques again performing best in all tests.

\subsection{Tests using real images}

Further to the synthetic tests, we have also tested the proposed technique against real medical images obtained from the Centre for Disease Control and Prevention (CDC) Public Health Image Library. The images of thin film Giemsa stained micrographs containing red blood cells were selected as a practical test, as the red blood cells are mostly circular (with some deformations in shape and size) and the images are of variable contrast and contain numerous overlapping cells along with various other image artefacts and noise. The selected test 


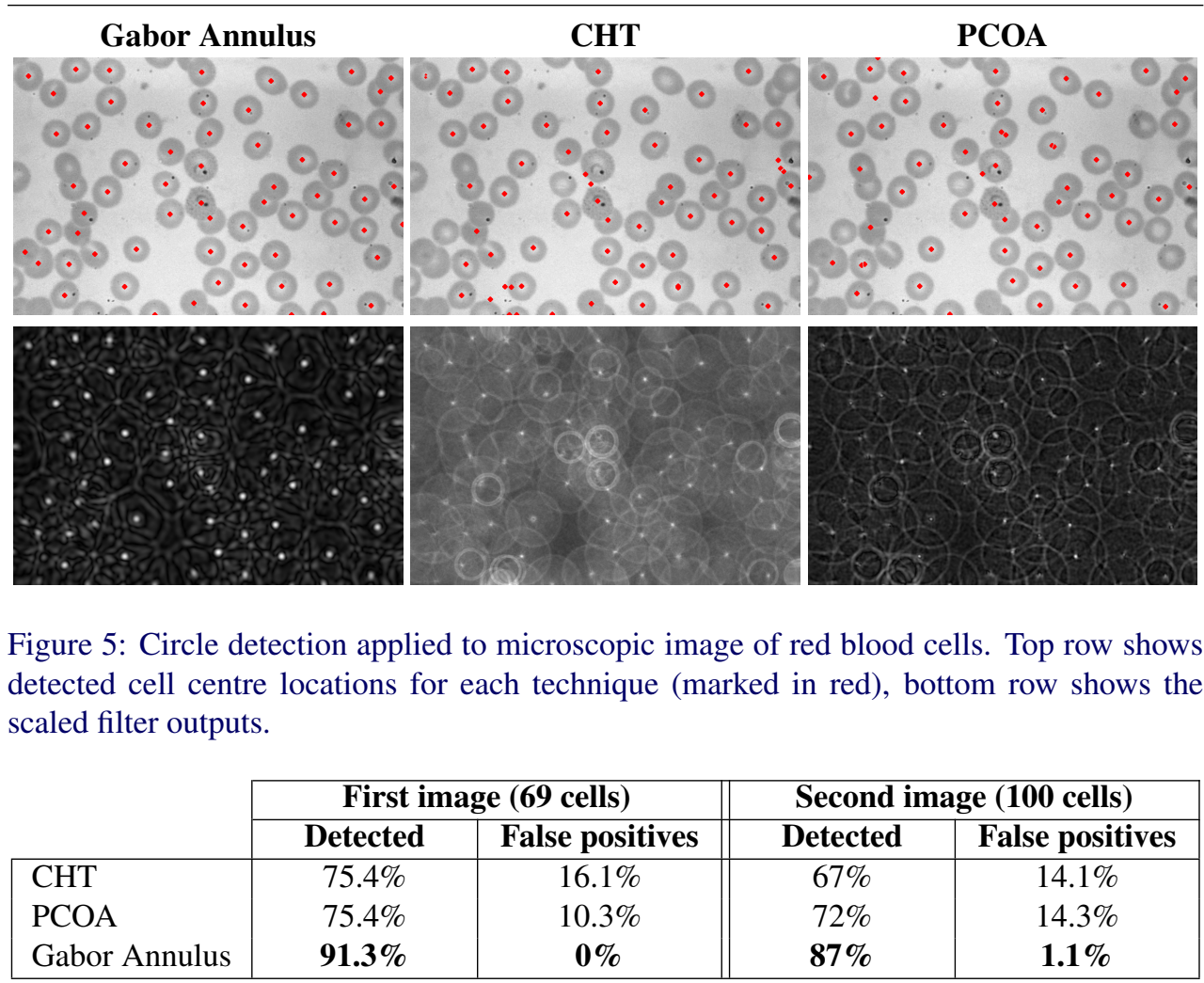

Table 2: Cell detection results using red blood cell microscopic images. The best results for each test are shown in bold. The circle detection techniques and test images used in these experiments are detailed in Section 3.2.

images can be seen in Figures 5 and 6.

The proposed technique was again compared to the standard CHT and Phase Coded Orientation Annulus (PCOA) methods. These techniques were applied to the required edge image (computed using the Sobel operator), however as demonstrated in the synthetic tests our proposed method is also capable of running directly against the image, without any preprocessing. We therefore take this approach in these tests. The red blood cells within the test images were measured and found to have a radius of around 30 pixels, therefore this radius was used to generate the filters for the $\mathrm{CHT}$ and proposed Gabor Annulus techniques. The PCOA technique uses a minimum and maximum radius, which were therefore set to 25 and 35 pixels respectively to cover deviations from the average cell size. The Gabor Annulus filters were generated using Equation 2 with the parameters $r_{0}=30, \sigma=30$ and $f_{0}=1 / 20$.

The filter output for each technique is scaled by the maximum response within the result, to give a result for each technique that lies in the range 0 to 1 . We then obtain the peaks corresponding to detected circles in the output by performing simple local thresholding. This is chosen rather than a global threshold in order to ensure that peaks in regions with a higher background response due to noise are also included. We use a local threshold window size of 20 pixels to calculate the mean value for the local neighbourhood, and threshold the values that have a response 0.25 higher than the local mean as cell locations. 

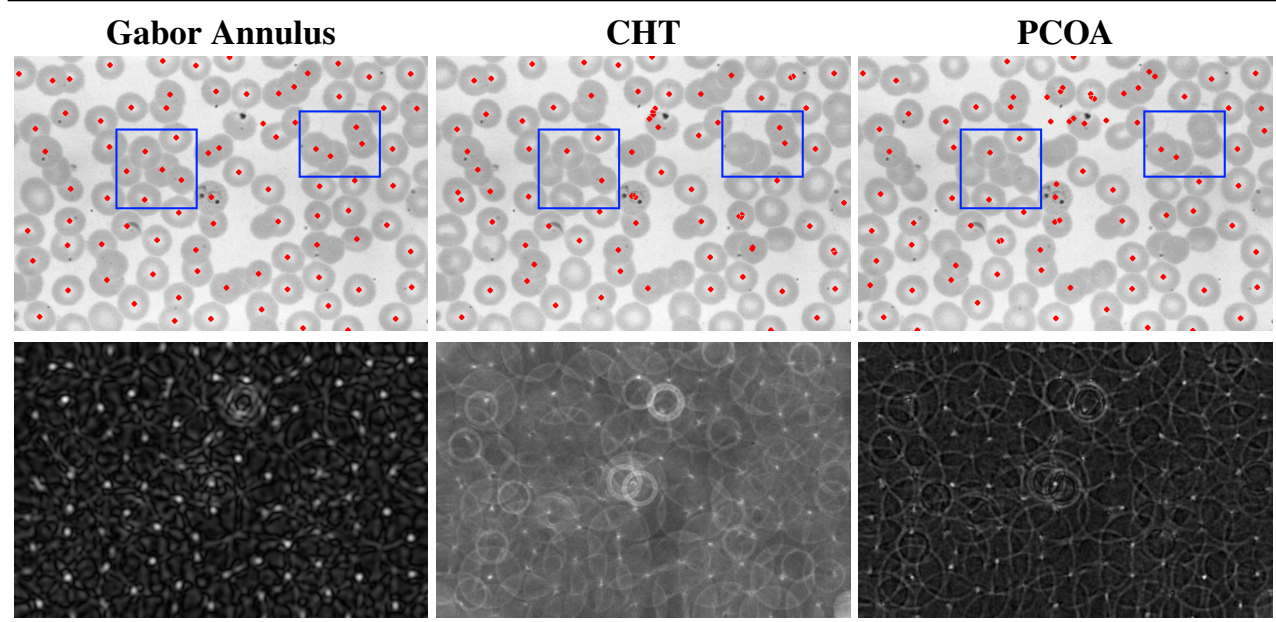

Figure 6: Circle detection applied to second microscopic image of red blood cells. Top row shows detected cell centre locations for each technique (marked in red), bottom row shows the scaled filter outputs. Example areas containing numerous overlapping cells are highlighted in blue rectangles for comparison of the techniques.

The resulting small blobs are eroded to a single centre location, and marked with red diamonds as detected cell centre locations in the top row of the result images, shown in Figures 5 and 6 . The first image contains fewer cells (69), and therefore less overlapping of individual cells, whilst the second image is a more thorough test as this includes a large amount of cells (100) with many overlaps.

The results of cell detection are presented in Table 2. It is clear that the proposed Gabor Annulus method has performed better in all cases, with $91.3 \%$ and $87 \%$ of the cells detected in the two test images. False positive rates (counted as the proportion of either incorrectly detected cell centres outside of an actual cell, or the extra incorrectly detected cells within a single cell) were also lowest for the proposed Gabor Annulus technique, at $0 \%$ and $1.1 \%$ for the two images respectively.

From the comparative result images shown in Figures 5 and 6 it is clear that the Gabor Annulus technique was better able to cope with overlapping cells, two regions containing such cells are highlighted in Figure 6 with blue rectangles.

\section{Conclusions}

We have presented a novel Gabor Annulus filter for use as a circle detector. This has been tested on both synthetic images and real microscopic images of circular red blood cells. In the synthetic tests the technique demonstrated higher peak to mean response ratios in all cases, clearer responses in high levels of noise, the ability to respond to overlapping circles with a lower background level response, and the ability to run directly on the image (i.e. without first performing edge detection) without affecting accuracy. This latter attribute makes the filter suitable for use in scenarios where clear edges are not necessarily present.

This was demonstrated in the real image tests using microscopic red blood cell images, where the proposed Gabor Annulus technique demonstrated the best detection rates and the 
lowest false positive rates. We therefore conclude that the proposed technique is a viable circle detector which offers advantages over traditional CHT and CHT based modifications, and has direct applications to real medical images.

In this work we have not addressed the topic of scale selection as we generated filters of the correct scale for the specific detection tasks. Investigating this topic with regard to the proposed Gabor Annulus filters will be important in order to assess the suitability of these filters for other tasks, and is the subject of ongoing work by the authors.

\section{References}

[1] T.J. Atherton and D.J. Kerbyson. Size invariant circle detection. Image and Vision Computing, 17(11):795-803, 1999. ISSN 0262-8856.

[2] F. Attneave. Symmetry, information, and memory for patterns. The American Journal of Psychology, pages 209-222, 1955. ISSN 0002-9556.

[3] O. Debeir, P. Van Ham, R. Kiss, and C. Decaestecker. Tracking of migrating cells under phase-contrast video microscopy with combined mean-shift processes. Medical Imaging, IEEE Transactions on, 24(6):697-711, 2005. ISSN 0278-0062.

[4] R.O. Duda and P.E. Hart. Use of the Hough transformation to detect lines and curves in pictures. Communications of the ACM, 15(1):11-15, 1972. ISSN 0001-0782.

[5] S. Eom, R. Bise, and T. Kanade. Detection of hematopoietic stem cells in microscopy images using a bank of ring filters. In Biomedical Imaging: From Nano to Macro, 2010 IEEE International Symposium on, pages 137-140. IEEE, 2010.

[6] B. Fang, W. Hsu, and M.L. Lee. On the accurate counting of tumor cells. Nanobioscience, IEEE Transactions on, 2(2):94-103, 2003. ISSN 1536-1241.

[7] Anil K. Jain and Farshid Farrokhnia. Unsupervised texture segmentation using gabor filters. Pattern Recognition, 24(12):1167 - 1186, 1991. ISSN 0031-3203. doi: DOI: 10.1016/0031-3203(91)90143-S.

[8] J.P. Jones and L.A. Palmer. An evaluation of the two-dimensional Gabor filter model of simple receptive fields in cat striate cortex. Journal of Neurophysiology, 58(6):1233, 1987. ISSN 0022-3077.

[9] N.N. Kachouie, P. Fieguth, J. Ramunas, and E. Jervis. Probabilistic model-based cell tracking. International Journal of Biomedical Imaging, 2006:1-10, 2006.

[10] D.J. Kerbyson and T.J. Atherton. Circle detection using Hough transform filters. In Image Processing and its Applications, 1995., Fifth International Conference on, pages 370-374. IET, 1995. ISBN 0852966423.

[11] N. Kharma, H. Moghnieh, J. Yao, Y.P. Guo, A. Abu-Baker, J. Laganiere, G. Rouleau, and M. Cheriet. Automatic segmentation of cells from microscopic imagery using ellipse detection. Image Processing, IET, 1(1):39-47, 2007. ISSN 1751-9659.

[12] T.S. Lee. Image representation using 2D Gabor wavelets. Pattern Analysis and Machine Intelligence, IEEE Transactions on, 18(10):959-971, 1996. ISSN 0162-8828. 
[13] C. Liu and H. Wechsler. Gabor feature based classification using the enhanced fisher linear discriminant model for face recognition. Image Processing, IEEE Transactions on, 11(4):467-476, 2002. ISSN 1057-7149.

[14] B.S. Manjunath and W.Y. Ma. Texture features for browsing and retrieval of image data. Pattern Analysis and Machine Intelligence, IEEE Transactions on, 18(8):837842, 1996. ISSN 0162-8828.

[15] E. Meijering, O. Dzyubachyk, I. Smal, and W.A. van Cappellen. Tracking in cell and developmental biology. In Seminars in cell \& developmental biology, volume 20, pages 894-902. Elsevier, 2009.

[16] L. O'Gorman, M.J. Sammon, and M. Seul. Practical algorithms for image analysis: description, examples, programs, and projects. Cambridge Univ Pr, 2008. ISBN 052188411X.

[17] B. Parvin, Q. Yang, J. Han, H. Chang, B. Rydberg, and M.H. Barcellos-Hoff. Iterative voting for inference of structural saliency and characterization of subcellular events. Image Processing, IEEE Transactions on, 16(3):615-623, 2007. ISSN 1057-7149.

[18] K. Riha and R. Benes. Circle detection in pulsative medical video sequence. In Signal Processing (ICSP), 2010 IEEE 10th International Conference on, pages 674-677. IEEE.

[19] O. Schmitt and M. Hasse. Radial symmetries based decomposition of cell clusters in binary and gray level images. Pattern Recognition, 41(6):1905-1923, 2008. ISSN 0031-3203.

[20] L. Shen and L. Bai. A review on Gabor wavelets for face recognition. Pattern Analysis \& Applications, 9(2):273-292, 2006. ISSN 1433-7541.

[21] L. Shen, L. Bai, and M. Fairhurst. Gabor wavelets and General Discriminant Analysis for face identification and verification. Image and Vision Computing, 25(5):553-563, 2007. ISSN 0262-8856.

[22] M. Smereka and I. Dulęba. Circular object detection using a modified hough transform. International Journal of Applied Mathematics and Computer Science, 18(1):85-91, 2008. ISSN 1641-876X.

[23] H.S. Wu, J. Berba, and J. Gil. Iterative thresholding for segmentation of cells from noisy images. Journal of microscopy, 197(3):296-304, 2000. ISSN 1365-2818.

[24] Jainguo Zhang, Tieniu Tan, and Li Ma. Invariant texture segmentation via circular gabor filters. Pattern Recognition, International Conference on, 2:20901, 2002. ISSN 1051-4651.

[25] Yanfang Zhang, Nongliang Sun, Yang Gao, and Maoyong Cao. A new eye location method based on ring gabor filter. In Automation and Logistics, 2008. ICAL 2008. IEEE International Conference on, pages 301 -305, sept. 2008. doi: 10.1109/ICAL. 2008.4636164 . 